\title{
Stabilization of hydrochlorothiazide nanocrystals using fibroin
}

\author{
Rani DHOLE 1 (D), Udaykumar PATIL 2 (D), Namdeo JADHAV $3 *$ (D)
}

1 Bharati Vidyapeeth College of Pharmacy, Kolhapur, Shivaji University, Maharashtra, India.

2 Department of Pharmaceutics, Bharati Vidyapeeth College of Pharmacy, Kolhapur, Shivaji University, Maharashtra, India.

3 Department of Pharmaceutics, Bharati Vidyapeeth College of Pharmacy, Kolhapur, Shivaji University, Maharashtra, India.

* Corresponding Author E-mail: nrjadhav18@rediffmail.com (N.J.); Tel. +91-231-263 72 86; Fax. +91-231-263 8833.

Received: 12 September 2018 / Revised: 31 May 2019 / Accepted: 31 May 2019

\begin{abstract}
Nanocrystals of the poorly water soluble drugs is a promising strategy. To stabilize the drug nanocrystals, stabilizers are usually used; however, the use of common stabilizers is limited by weak stabilization effect and toxicological concerns for long-term treatment. The present work was aimed to investigate the potential of natural silk protein as novel stabilizer for nanocrystal of hydrochlorothiazide, which was a model drug. The nanocrystals of drug with hydrophobic protein stabilizer were prepared by antisolvent precipitation method. Prepared nanocrystals were evaluated for parameters like particle size, zeta potential, DSC, XRD, \%crystallinity, SEM, drug content and in vitro dissolution test. Stabilization efficiency of nanocrystals was assessed by their \% crystallinity for 3 months. Optimized batch R2 was shown smaller particle size, highest drug content and drug dissolution. Nanocrystals were shown the extended release due to coat of fibroin around the hydrochlorothiazide. From the \% crystallinity study it was found that there was not significant change in the nanocrystals prepared using fibroin. So, it was concluded that the fibroin is good stabilizer for drug nanocrystals.
\end{abstract}

KEYWORDS: Crystallinity; fibroin; nanocrystal; stabilization.

\section{INTRODUCTION}

Nanocrystals are particles having at least one dimension smaller than $100 \mathrm{~nm}$ and composed of atoms in either a single or polycrystalline arrangement. Nanonization is most widely used technique to overcome the dissolution issues of poorly soluble drugs by reduction in particle size and increased surface area [1]. The increase in specific surface area assumes significance in catalysis, thus nanoscale materials can be useful as powerful catalysts. Nanocrystals can be prepared by top-down (high pressure homogenization, milling) technique, bottom-up (precipitation, supercritical fluid) and combination technique $[2,3]$. Here we used the antisolvent precipitation followed by spray drying method.

Nanocrystals have the stability problems like the massive surface area of nanocrystals which results in sufficiently high free energy or surface charge. Free energy might cause attraction or agglomeration and the other cause is the ostwald ripening (recrystallization) The prevention of aggregation may be achieved by the addition of surface active agent including ionic surfactants, non-ionic surfactants, and polymers which can provide an electrostatic and steric repulsion between the nanocrystals. Thus, development of stable nanocrystals of poorly soluble drug \& ensure its feasibility is a pre-requisite. Nanocrystal technology is used to solubility enhancement and also used for narrow therapeutic window where drug gets absorbed. A drug nanocrystal as carriers for biomolecular delivery is another trend since drug nanocrystals could enhance the intracellular uptake of macromolecules and protect them from denaturation [4]. Nanocrystals can be stabilized by both steric and electrostatic phenomenon [5]. After the disulphide bonds buried inside the denatured protein, other buried hydrophobic residues (disulphide bonds) were exposed on the surface of biopolymers by increasing the area of hydrophobic regions and then enhancing affinity of proteins for drug surface. Thus, effective steric stabilization was produced by the absorbance of protein onto the drug particles [6]. In order to avoid agglomeration and ostwald ripening we propose the use of fibroin (insoluble natural protein) produced by silkworm Bombyx mori, as a stabilizer to improve the stability of nanocrystals. Silk in its raw state consists

How to cite this article: Dhole R, Patil U, Jadhav N. Stabilization of hydrochlorothiazide nanocrystals using fibroin. J Res Pharm. 2019; 23(6): 9971008. 
of two main proteins, sericin and fibroin, with a glue-like layer of sericin coating two singular filaments of fibroin called brin. The fibroin protein consists of layers of antiparallel beta sheets [7]. Considering biocompatibility and diversity in the selection of monomers, amino acid \& proteins are excellentcandidates for this purpose [12]. Literature was revealed that food proteins are widely used in formulated foods because they have high nutritional values and are generally recognized as safe. Therefore, we proposed the use of natural protein such as fibroin. Silk fibroin is abundantly available in nature; lot of work going on fibroin because of its own properties such as biocompatibility, hydrophobicity, impressive mechanical strength, etc. One more advantage is that silk is natural source of protein. Objectives of the work are extraction and isolation of silk fibroin, design and development of drug nanocrystals using fibroin, stability assessment for nanocrystals. Stabilization of nanocrystals is the today's need and the proposed work will be helpful in nanotechnology arena.

\section{RESULTS AND DISCUSSION}

\subsection{Percentage yield of spray drying product}

The percentage yield of spray drying obtained with optimized parameter was in the range of $62-80 \%$. Yield of all spray dried formulation batches shown in Table 1.

Table 1. Spray dried product yield of all formulation batches.

\begin{tabular}{ccc}
\hline Sr. No. & Batch code & $\begin{array}{c}\% \text { process } \\
\text { yield }\end{array}$ \\
\hline 1 & R1 & 65 \\
2 & R2 & 80 \\
3 & R3 & 74 \\
4 & R4 & 62 \\
\hline
\end{tabular}

\subsection{FTIR analysis}

FTIR studies revealed that the fundamental peaks of Hydrochlorothiazide (HCTZ) retained in the optimized formulation and indicated the absence of any chemical interaction between Hydrochlorothiazide and fibroin. Thus, fibroin used in the Hydrochlorothiazide nanocrystal formulation. FTIR spectrum of fibroin, HCTZ, physical mixture of drug and fibroin, optimized batch R2 of HCTZ nanocrystal shown in Figure 1a, b, c and d respectively. From the FTIR studies, it shows that the principal peak at $3050 \mathrm{~cm}^{-1}$ of the pure drug Hydrochlorothiazide retained in the optimized batches, which was almost identical.

\subsection{X-Ray diffraction study}

X- ray diffraction spectra of fibroin, HCTZ, physical mixture of both has carried out. Overlain XRD of HCTZ, fibroin and physical mixture and four different batches of nanocrystals that are R1 (1:0.25), R2 (1:0.5), R3 (1:0.75) and R4 (1:1) were also carried to study the crystallinity and possible polymorphic transformations in the formulation showed in Figure $2 \mathrm{a}, \mathrm{b}, \mathrm{c}, \mathrm{d}, \mathrm{e}, \mathrm{f}, \mathrm{g}$, h respectively. X-ray diffraction used to analyze potential changes in the crystalline nature of Hydrochlorothiazide crystals.

XRD of HCTZ and fibroin-HCTZ nanocrystal optimized batch R2 compared to detect the crystallinity and polymorphic transformation. The diffraction spectrum of pure HCTZ showed the drug was of crystalline nature as indicated by numerous, relative sharp and distinct peaks at a diffraction angle of $2 \theta$ shown in Figure $2 \mathrm{~b}$. The nanocrystals of optimized batch R2 characterized by same intensity of the diffraction peak, that is at $30^{\circ} 2 \theta$ intense peak was seen with intensity of 900 of HCTZ shown in Figure $2 \mathrm{f}$. This clearly indicates the insignificant reduction in the crystallinity of the precipitated HCTZ nanocrystals, the ordered crystals were majority, and the crystalline state would contribute to the lower drug loading capacity. The persistent sharp confirmed that HCTZ existed in the crystalline state in the nanocrystals form. 
A

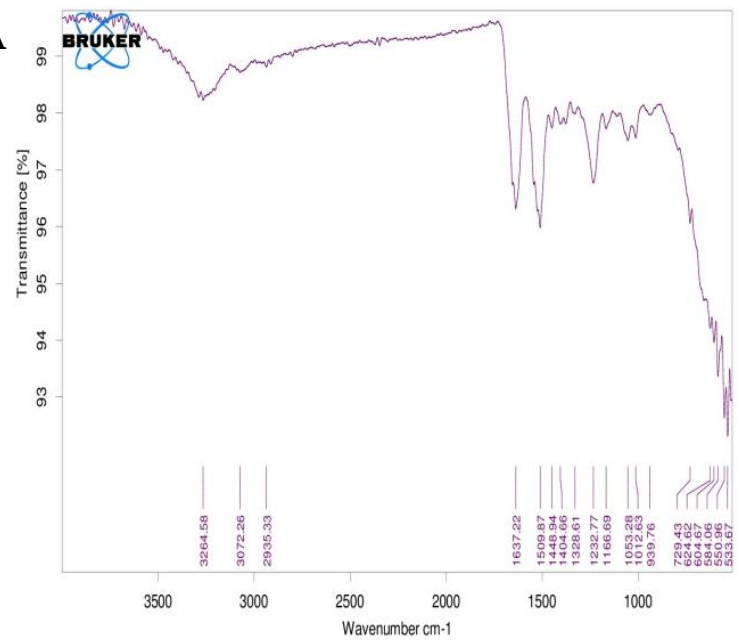

B

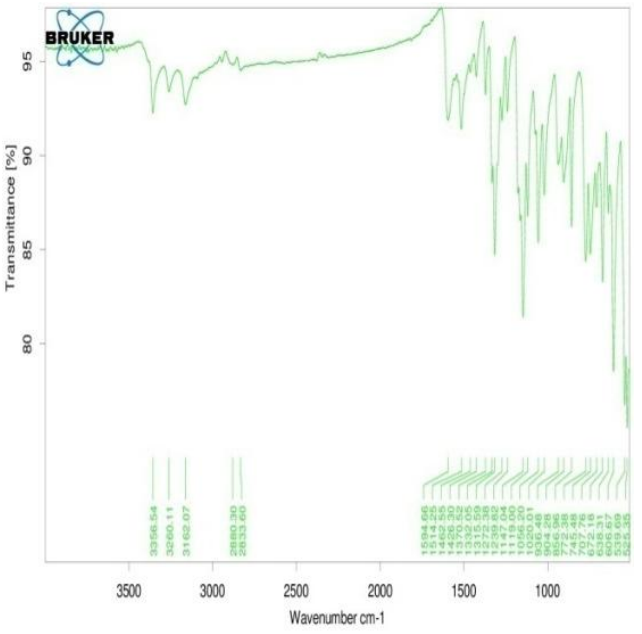

B

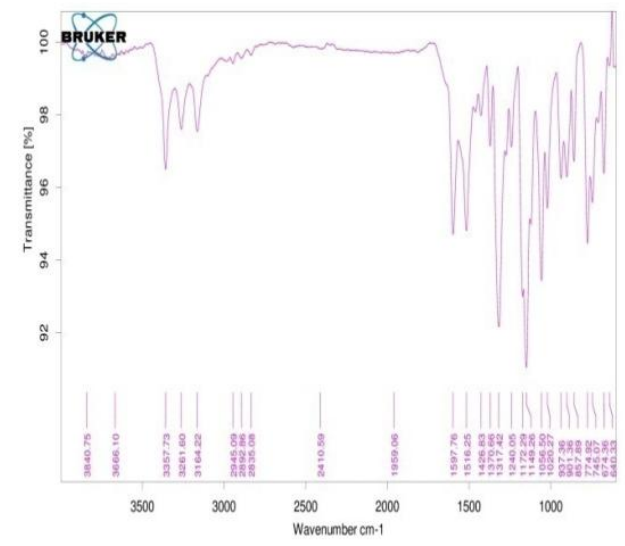

D $\stackrel{\circ}{-}$ BRUKER

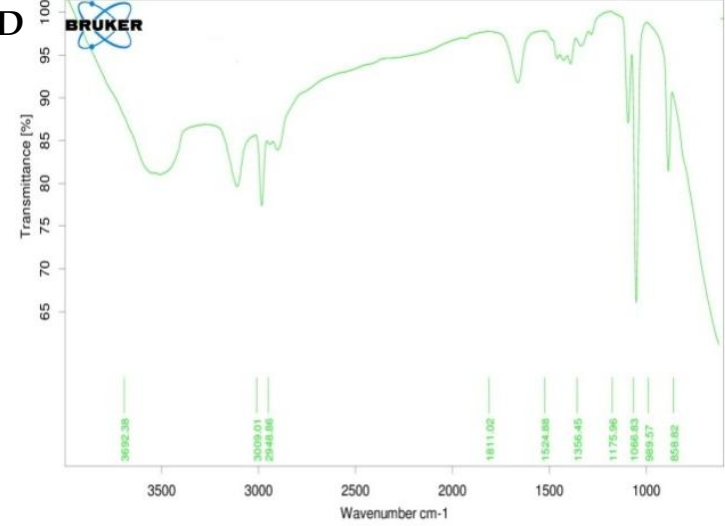

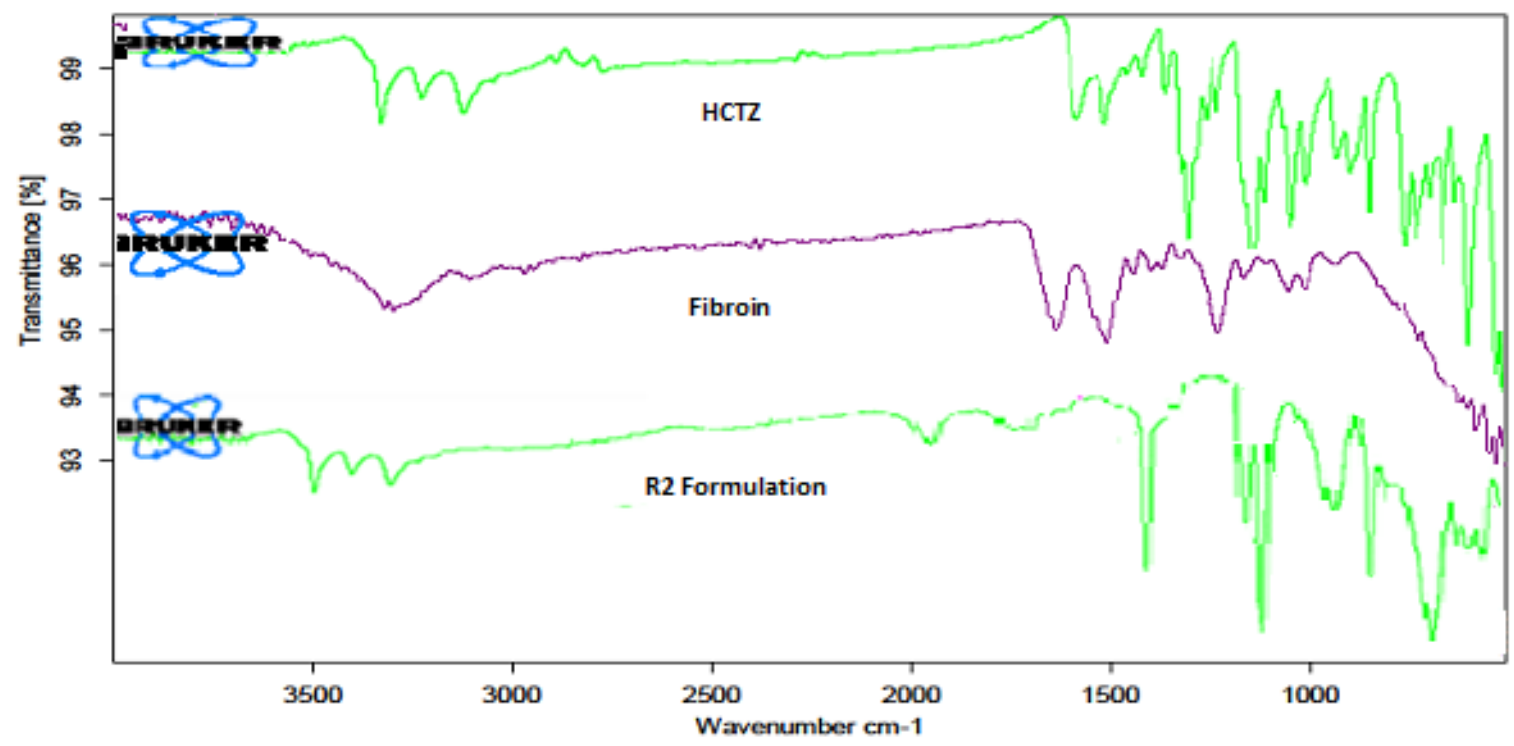

Figure 1. FTIR of A. Fibroin, B. Drug (HCTZ), C. Physical mixture of drug and fibroin, D. Optimized batch R2 of HCTZ nanocrystal. 
A

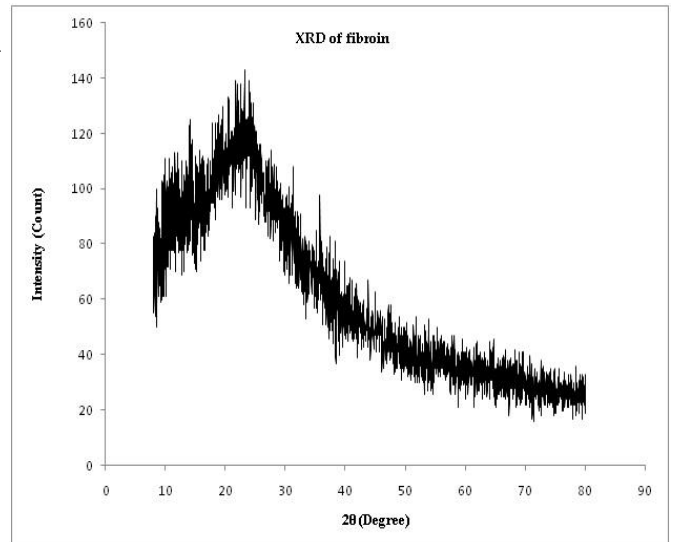

C

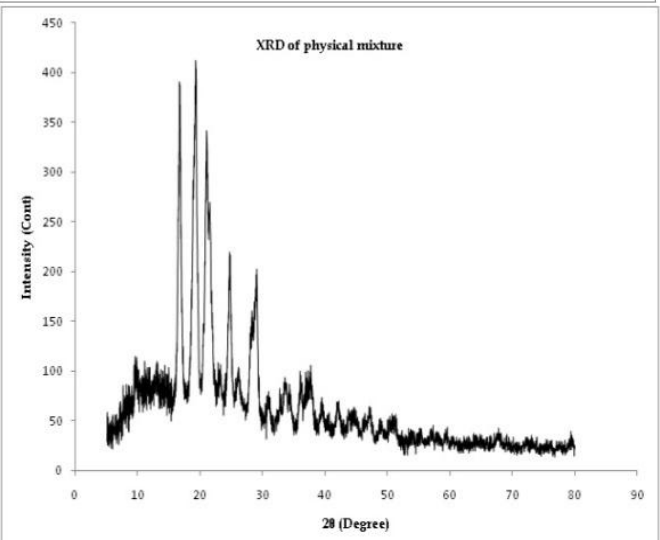

E

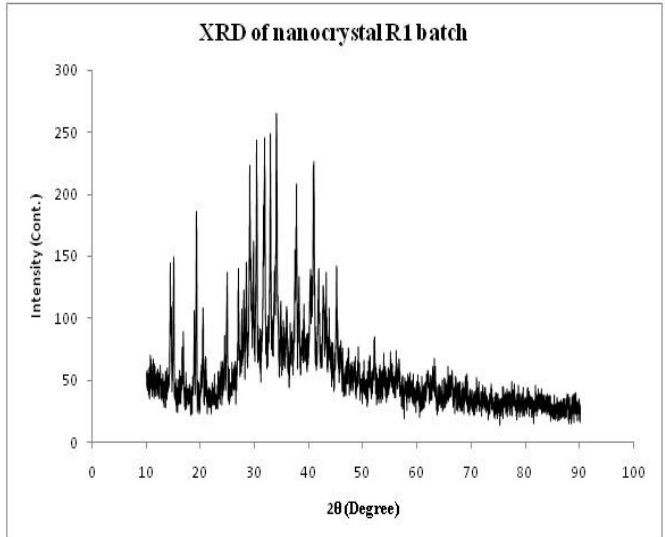

G

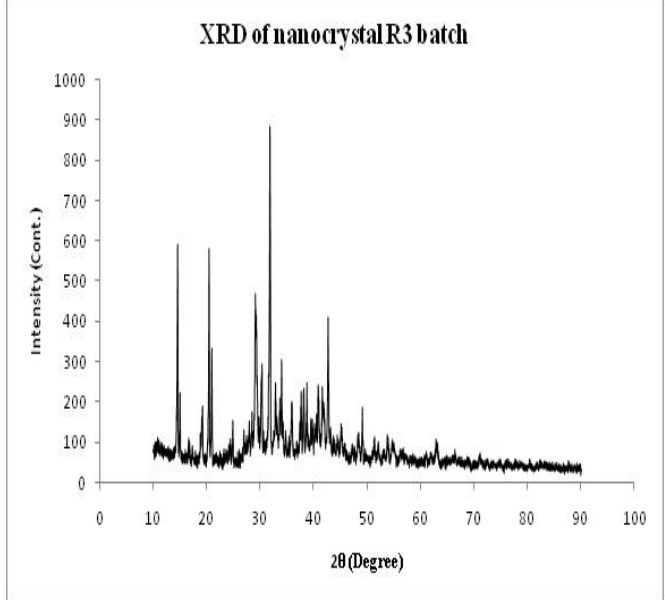

B

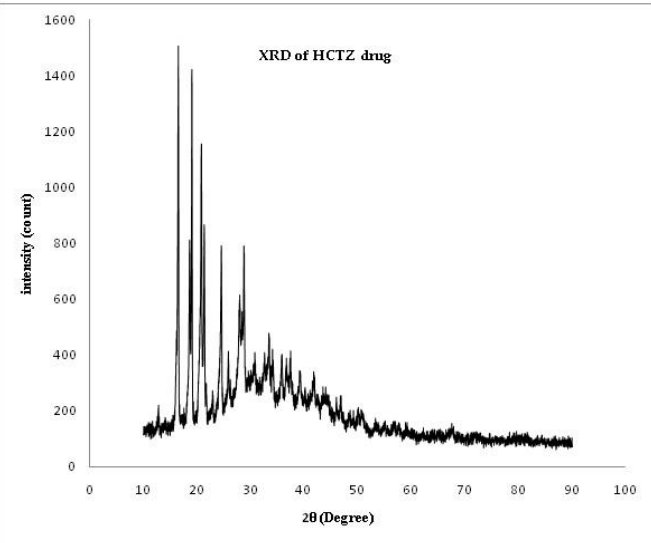

D

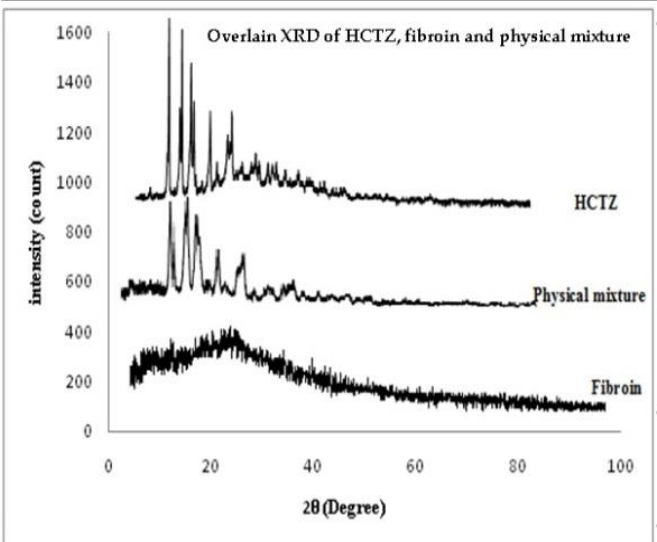

$\mathbf{F}$

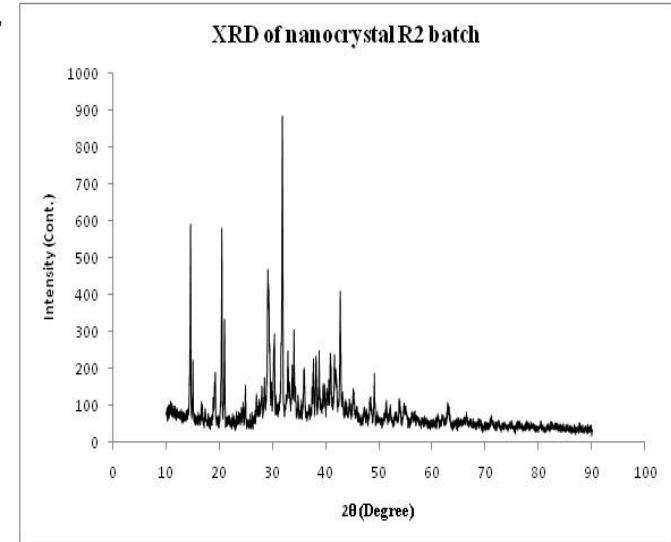

$\mathbf{H}$

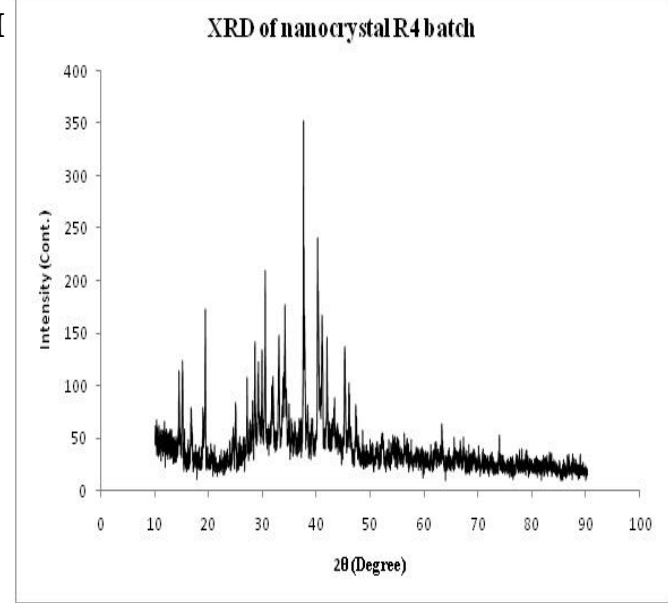

Figure 2. X ray of a. Fibroin, b. Drug HCTZ, c. Physical mixture, d. Overlain of HCTZ, fibroin and physical mixture, e. Nanocrystal formulation batch R1, f. Nanocrystal formulation batch R2, g. Nanocrystal formulation batch R3, h. Nanocrystal formulation batch R4. 


\subsection{Particle size analysis}

Particle size and the polydispersity index (PDI) of all the formulations were measured by dynamic light scattering (DLS; Malvern Zetasizer, Nano-ZS90, UK). It found that the smallest particle size observed in batch $\mathrm{R} 2$ as compared to other formulations as shown in Table 2. Particle size analyses of all batches shown in Figure 3.

Table 2. Evaluations of HCTZ nanocrystals.

\begin{tabular}{cccccc}
\hline & \multicolumn{5}{c}{ Evaluation parameters } \\
\cline { 2 - 6 } Batch Code & Particle Size & PDI & $\begin{array}{c}\text { Zeta } \\
\text { potential } \\
(\mathbf{m V})\end{array}$ & $\begin{array}{c}\% \\
\text { Crystallinity }\end{array}$ & $\begin{array}{c}\text { \%Drug } \\
\text { content }\end{array}$ \\
\cline { 2 - 6 } & 472.4 & 0.776 & -6.01 & 15.87 & 88.50 \\
R1 & 251.8 & 0.737 & -18.8 & 31.75 & 95.56 \\
R3 & 377.6 & 0.683 & -16.3 & 12.62 & 90.83 \\
R4 & 401.4 & 0.466 & -4.11 & 15.86 & 81.25 \\
\hline
\end{tabular}

A

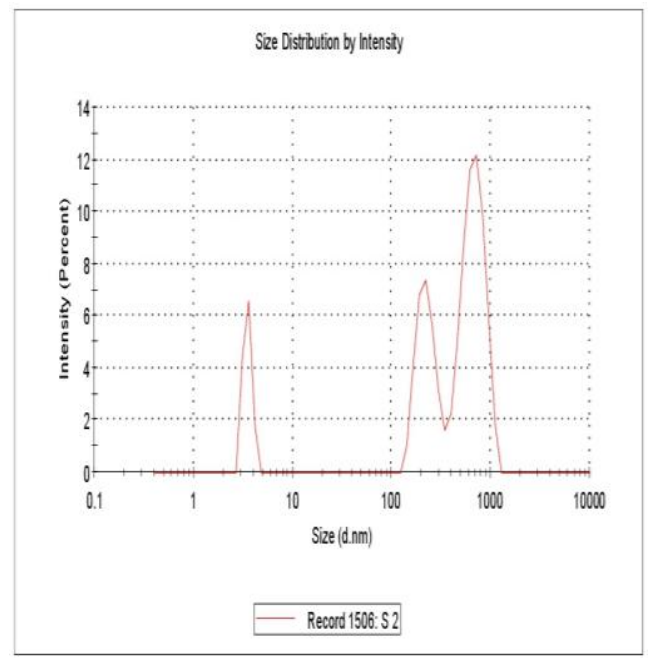

C

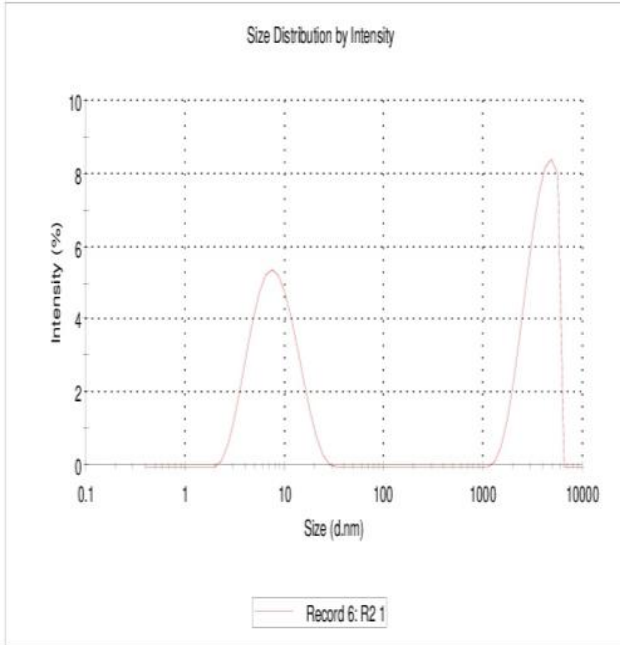

B

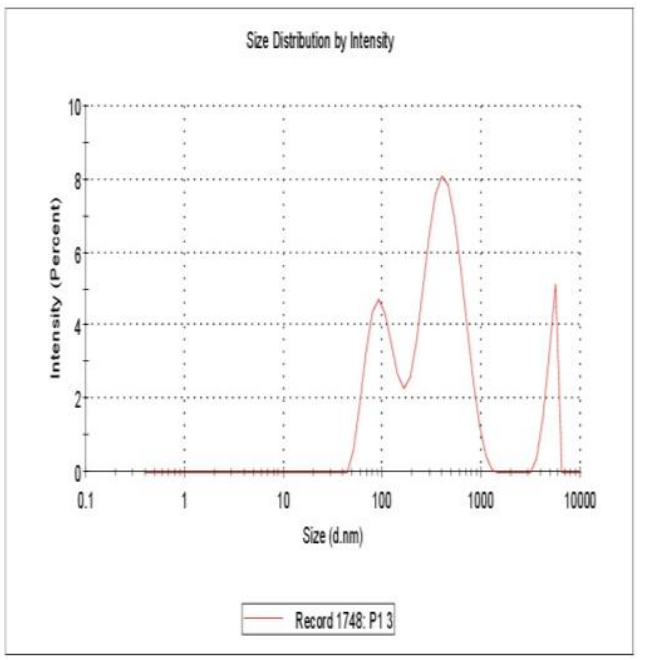

D

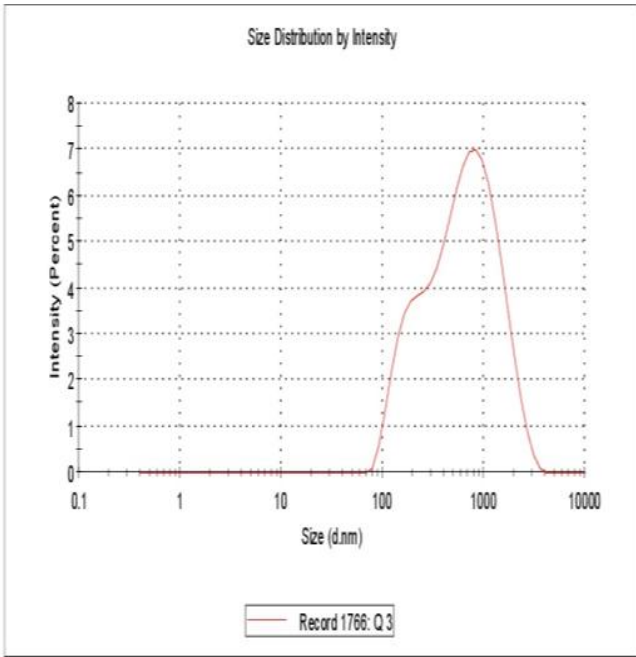

Figure 3. Particle size analysis of nanocrystal formulations A. Batch R1, B. Batch R2, C. Batch R3, D. Batch R4. 


\subsection{Zeta potential analysis}

The Malvern Zetasizer Nano ZS (Malvern Instruments, UK) measured the zeta potential of the samples. [11] The zeta potential results of the nanocrystal of all formulations shown in Table 2. Zeta potential value of all the batches was found to be in between -4.11 to $-18.8 \mathrm{mV}$, optimized batch shows $-18.8 \mathrm{mV}$ mean zeta potential which means optimized batch have more stability than other batches. Zeta potential analysis of all batches was shown in figure 4 .
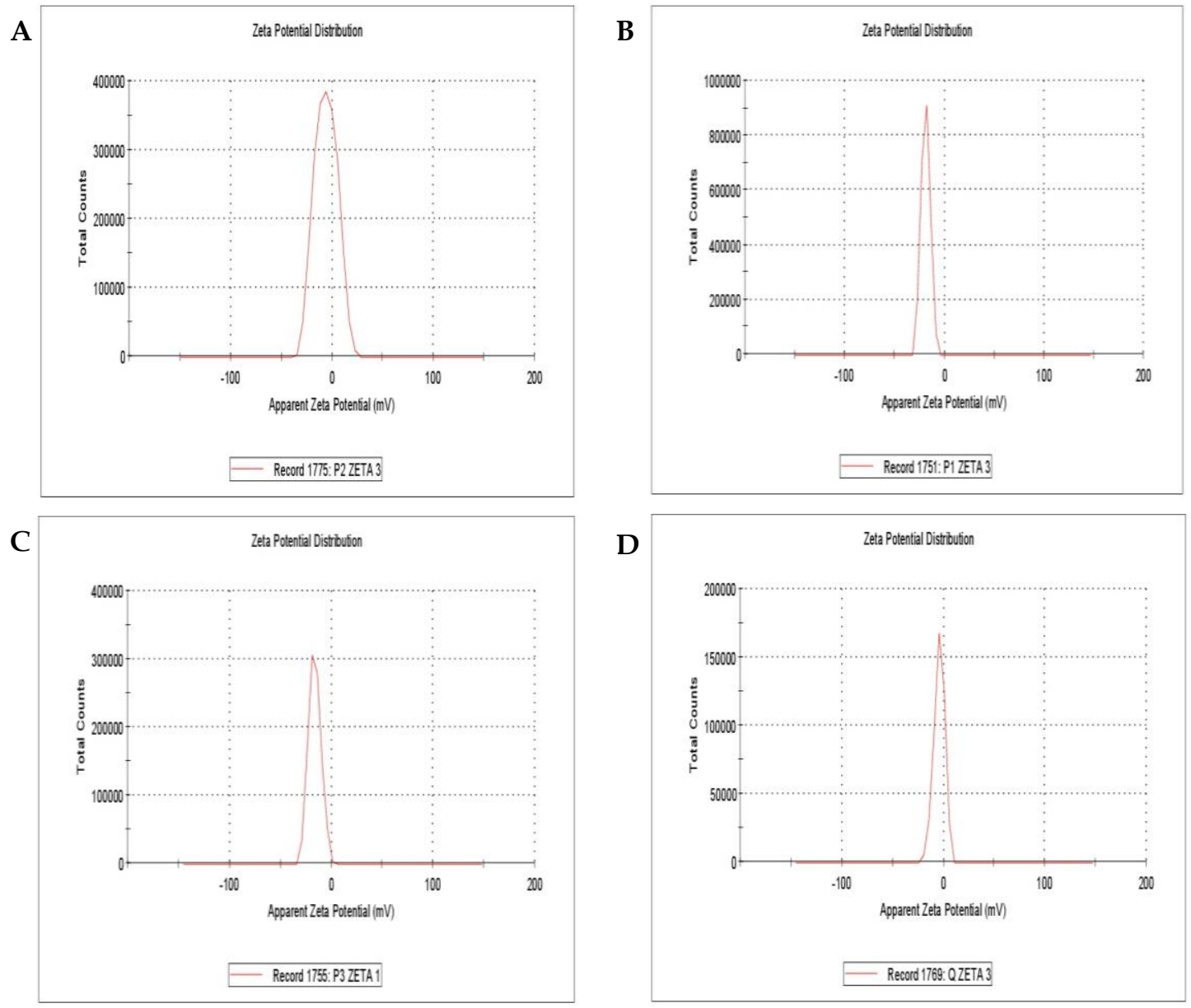

Figure 4. Zeta potential of nanocrystals formulations a. Batch R1, b. Batch R2, c. Batch R3, d. Batch R4.

\subsection{Differential scanning calorimetry}

DSC of four different batches of nanocrystals R1 (1:0.25), R2 (1:0.5), R3 (1:0.75) and R4 (1:1) were carried to analyze the polymorphic transformation, the energy required to melt the sample $(\Delta \mathrm{H})$.

DSC thermogram of four different formulations shows the sharp endothermic peak at $270{ }^{\circ} \mathrm{C}$. It means that there was no change in the crystalline nature of the drug.

Differential Scanning Calorimetry (DSC) used to investigate and predict the changes in the crystalline form of the drug. Figure 5a, b, c, d shows the DSC results of the fibroin, HCTZ, optimized nanocrystals formulation batch R2, and overlain DSC of drug and optimized batch R2. The pure drug exhibited a large and sharp endothermic peak at $269.57^{\circ} \mathrm{C}$ indicating the melting point. DSC thermogram of formulation R2 showed an endothermic peak at $270^{\circ} \mathrm{C}$ (Figure 5c) ascribed to the melting of Hydrochlorothiazide indicated the slight change in the crystalline nature. However, no additional peaks found to demonstrate the significant changes in the melting characteristics of Hydrochlorothiazide in the formulation indicating no polymorphic changes in the Hydrochlorothiazide. The peaks were found to be nearly identical, with a calculated enthalpy $(\Delta \mathrm{H})$ of pure drug and R2 were around $-144.40 \mathrm{~J} / \mathrm{g},-98.23 \mathrm{~J} / \mathrm{g}$, respectively. 
A

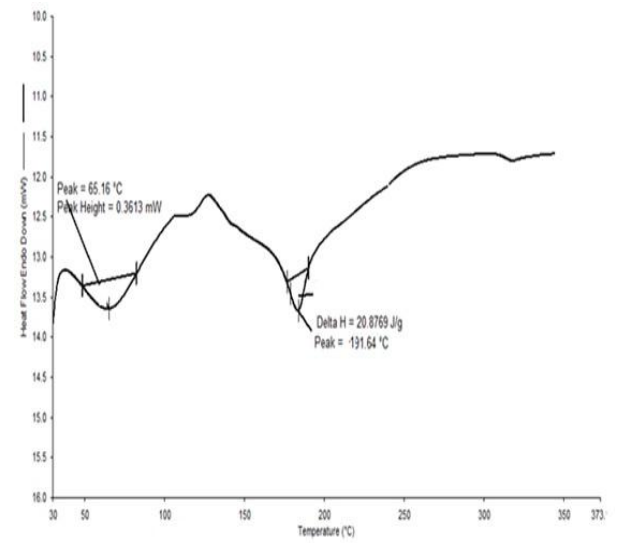

C

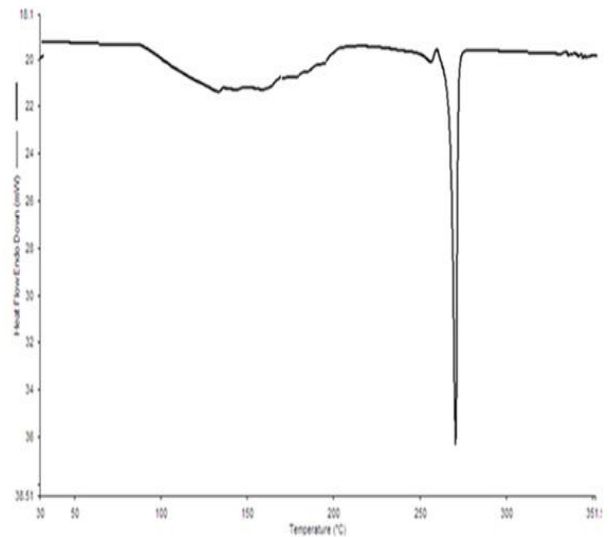

B

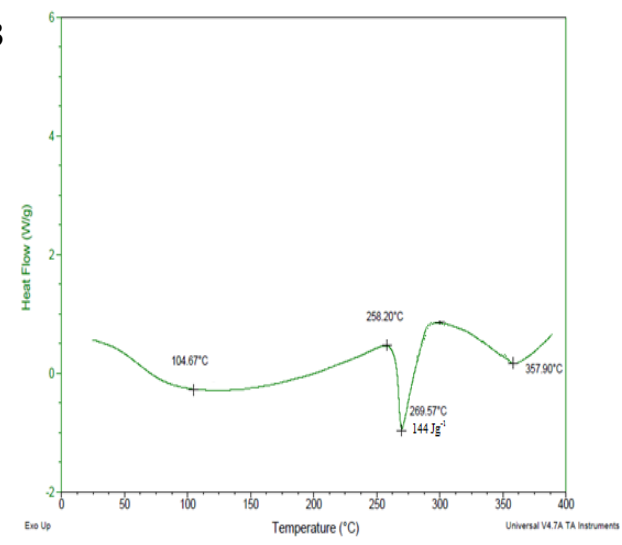

D

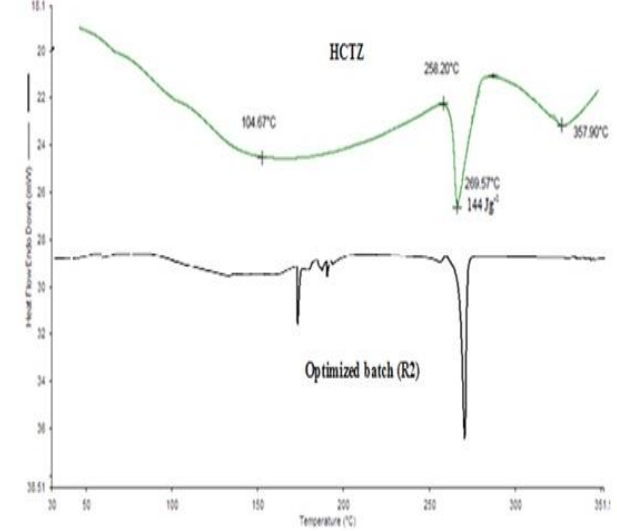

Figure 5. DSC of a. Fibroin, b. Drug HCTZ, c. Optimized nanocrystals formulation batch R2, d. OverlainDSC of drug and optimized batch R2.

\subsection{Percentage crystallinity}

Percent crystallinity of formulated nanocrystal calculated from the equation:

$$
\% \text { Crystallinity }=\frac{\delta H m S D}{\delta H m D r u g \times W} \times 100
$$

Where, melting enthalpy of nanocrystals was taken as $\delta \mathrm{HmSD}$, melting enthalpy of the drug was taken as $\delta H m D r u g$, and $W$ was taken as the weight fraction of drug in fibroin. Percent crystallinity of four formulated nanocrystals found between 12.62 and 31.75, which enlisted in Table 2. R2 batch shows highest crystallinity that is 31.75 , which indicates the R2 batch has good crystallinity.

\subsection{Drug content determination}

The drug content of spray dried nanocrystals batches was shown in Table 2. Lesser loss of drug content of optimized batch during spray drying resulted in the good recovery of nanocrystals. Drug content of all batches found 81.25 to 95.56 .

\subsection{In vitro dissolution study of nanocrystal formulations}

The in vitro dissolution profile of all nanocrystal formulations carried out in dissolution media $0.1 \mathrm{~N}$ $\mathrm{HCl}$ and phosphate buffer $\mathrm{pH}$ 6.8, which shown in Figure 6. Pure drug HCTZ shows $90 \%$ dissolution within $2 \mathrm{hr}$. Nanocrystal formulation batch R2 was showed higher \% dissolution of the drug as compared with that of all remaining batches $(\mathrm{R} 1=83.73 \%, \mathrm{R} 3=84.05 \%, \mathrm{R} 4=81.02 \%)$. The percentage dissolution of optimized batch $\mathrm{R} 2$ observed was $86.24 \%$. Within $12 \mathrm{~h}$, maximum dissolution took place.

From dissolution profile it was observed that capsule containing HCTZ silk fibroin nanocrystals (batch R2) was placed in dissolution medium it first gets swells followed by drug dissolution over an extended period of time from the matrix formed by silk fibroin. It has concluded that silk fibroin imparts good matrix integrity as for extended drug dissolution. From the dissolution studies of the formulations, formulation of batch HCTZ 
fibroin nanocrystal filled capsule has showed better drug dissolution with extended dissolution and good matrix integrity throughout dissolution period. Hence, formulation R2 considered as the best formulation.

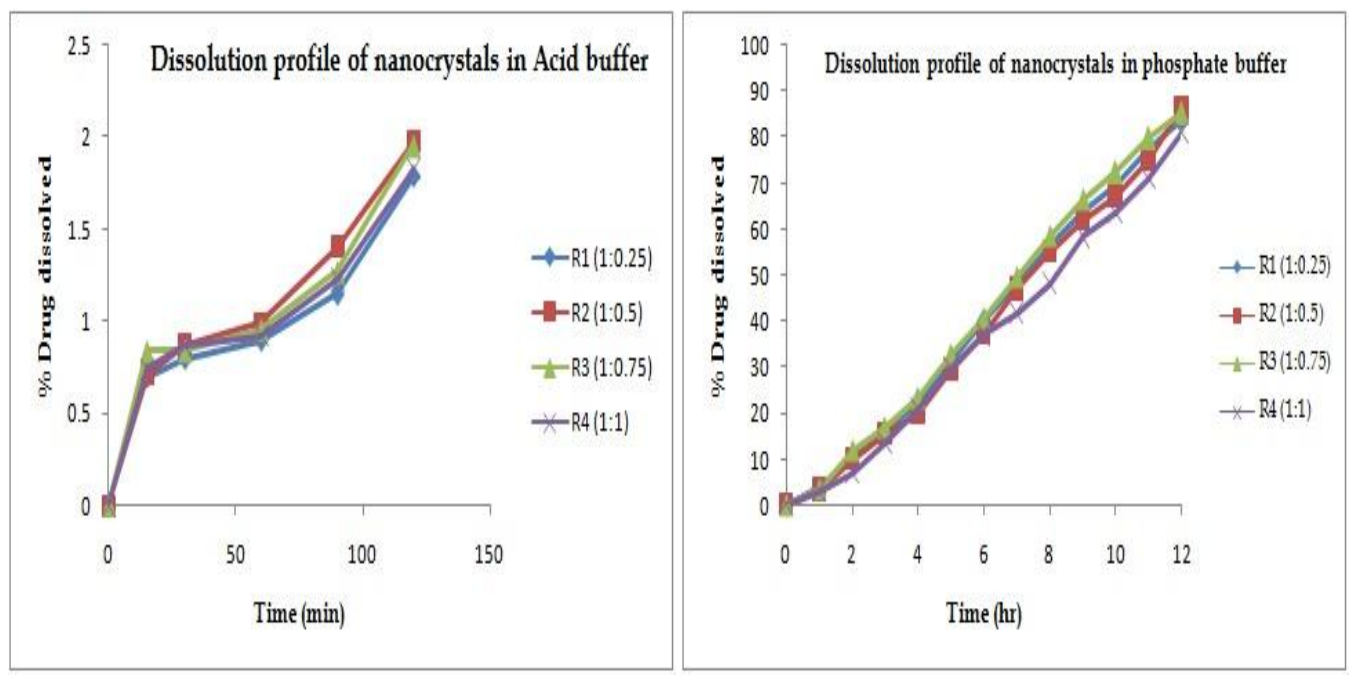

Figure 6. Dissolution profiles of HCTZ nanocrystals in acid buffer and phosphate buffer.

\subsection{Scanning electron microscopy}

Surface morphology of the formed crystals was determined by using SEM and it found crystalline. Crystalline nature of all the formulations remains with the slight change in crystallinity. The SEM images of the HCTZ nanocrystals presented in Figure 7. The nanocrystal has found to be spherical in shape with a narrow particle size distribution.
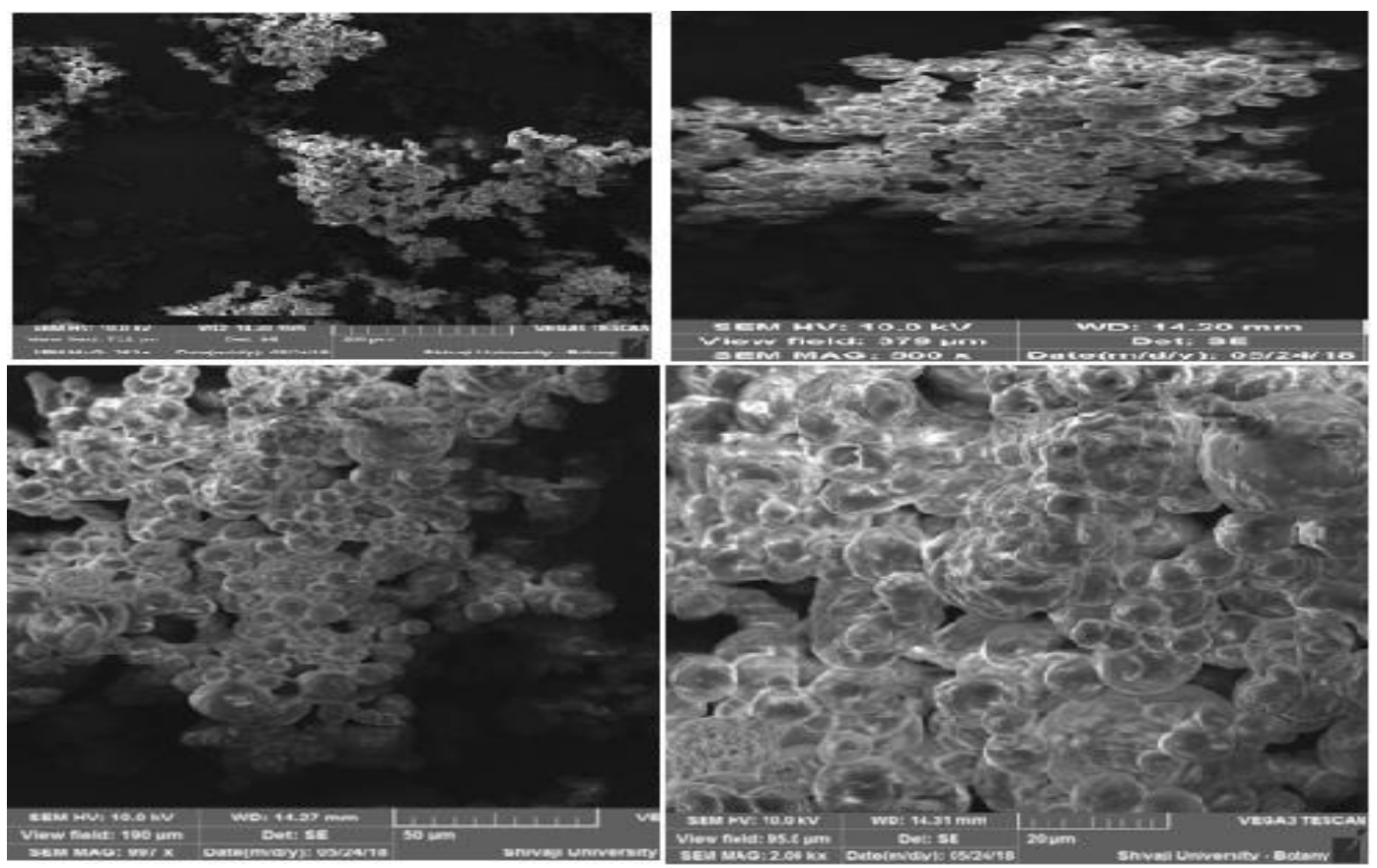

Figure 7. SEM images of optimized batch R2 at $10 \mathrm{kV} \times 250,500,1000$, and 2000.

\subsection{Stability study}

The filled capsules of nanocrystals kept for stability study at two temperature conditions that is $2-8^{\circ} \mathrm{C}$ and at RT. It should be kept for 3 months and analyzed periodically for 30, 60, 90 days.

The stability of hydrochlorothiazide loaded nanocrystals in terms of drug content, particle size distribution, zeta potential, percentage crystallinity has monitored for 3 months at $2-8^{\circ} \mathrm{C}$ and $\mathrm{RT}\left(25-30^{\circ} \mathrm{C}\right)$. 
The nanocrystals showed physical stability for a period of 3 months at refrigerated conditions. The particle size, drug content, zeta potential, percentage crystallinity of the nanocrystals at different time interval shown in table 3.

Table 3. Stability data of optimized batch.

\begin{tabular}{lcccc}
\hline Time & Initial & 1 month & 2 month & 3 month \\
\hline Drug content (\%) (2-8 $\left.{ }^{\circ} \mathbf{C}\right)$ & $100.60 \pm 0.5$ & $100.55 \pm 0.48$ & $100.41 \pm 0.34$ & $100.35 \pm 0.44$ \\
Drug content (\%) (RT) & $101 \pm 0.45$ & $101 \pm 0.26$ & $100.96 \pm 0.62$ & $100.50 \pm 0.49$ \\
Particle size (nm) (2-8 $\left.{ }^{\circ} \mathbf{C}\right)$ & $251.14 \pm 12.6$ & $268.67 \pm 10.2$ & $270.16 \pm 15.3$ & $271.6 \pm 13.9$ \\
Particle size (nm) (RT) & $265.14 \pm 8.3$ & $280.3 \pm 13.5$ & $288.6 \pm 14.2$ & $294.1 \pm 8.9$ \\
Zeta potential (2-8 $\left.{ }^{\circ} \mathbf{C}\right)$ & -18.8 & -18.8 & -18.9 & -18.8 \\
\% Crystallinity (RT) & 31.75 & 31.75 & 32.09 & 32.09 \\
\hline
\end{tabular}

At room temperature, Particle size was increased and drug content found decreased. It found that no significant difference observed in the particle size and drug content of nanocrystals (figure 8) after 3 months at refrigerated conditions indicating its suitability for storage at $2-8^{\circ} \mathrm{C}$.

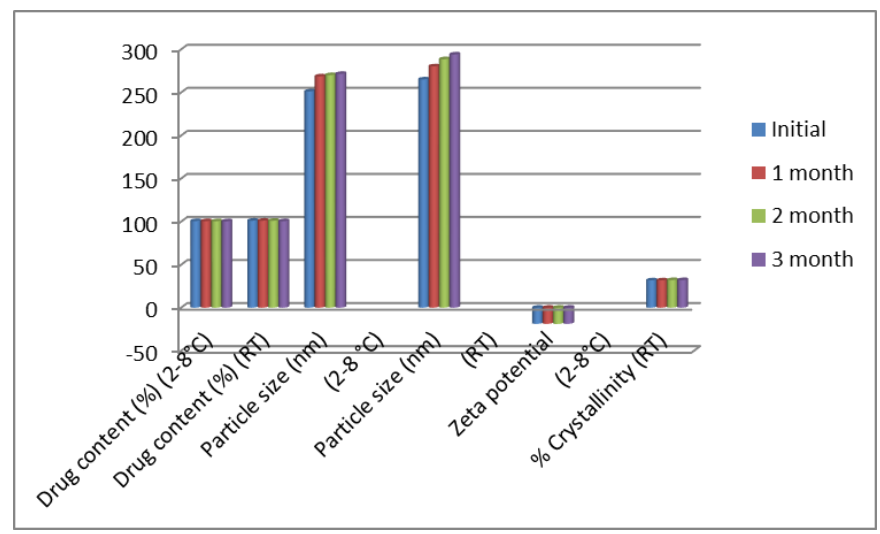

Figure 8. Stability profile of optimized batch of nanocrystals for different parameters.

Zeta potential, drug content, crystallinity remains constant at $2-8^{\circ} \mathrm{C}$ indicates that there was no significant difference observed. Percentage crystallinity of nanocrystals has slightly increased but not any noteworthy difference after three months storage (Figure 8).

\section{CONCLUSION}

The present study was an attempt to formulate Nanocrystal of poorly water soluble drug, Hydrochlorothiazide having low solubility. The objective was to stabilize the nanocrystals to minimize the aggregation, crystal growth by reducing surface charge and free energy.

The Nanocrystal formulation of HCTZ with the smaller particle size can be effectively produced by the antisolvent precipitation method. Thus Nanocrystal approach may be a promising approach for other Class II and IV drugs. The stabilized nanocrystals of hydrochlorothiazide were successfully formulated using hydrophobic fibroin protein. Thus, the present fibroin protein has great future for its greater stability and solubilisation property, when combined with hydrophilic protein.

\section{MATERIALS AND METHODS}

Hydrochlorothiazide was obtained as gift sample from Abbott Laboratories, Goa, India. Silk cocoons were collected from Government Silk Processing Center, Islampur, Sangli. All other chemicals used were of analytical grade.

\subsection{Preparation of nanocrystals}

Hydrochlorothiazide nanocrystals were prepared by antisolvent precipitation method [8]. Four different batches of nanocrystals were prepared. Drug : polymer ratio was taken in four different combinations like 1:0.25, 1:0.5, 1:0.75 and 1:1.. $60 \mathrm{mg}$ of the drug was dissolved in organic solvent \& in proportion of $0.25,0.5$, 0.75, 1 fibroin dissoved in aqueous Antisolent. The antisolvent was cooled to below $5^{\circ} \mathrm{C}$ in an ice-water bath. 
Organic solution was added dropwise into $50 \mathrm{ml}$ of the pre-cooled fibroin in antisolvent at a stirring speed of $1000 \mathrm{rpm}$. Prepared suspension that is the nanoparticles were then spray dried at $120^{\circ} \mathrm{C}$ temperature \& $30 \mu \mathrm{l} / \mathrm{min}$ aspiration speed. [10] Obtained nanocrystals stored in tightly closed container and analysed for various parameters. Literature has been revealed that the nanocrystals having wide applications [13].

\subsection{Characterization of nanocrystals}

\subsubsection{Percentage yield of spray drying process}

The amount of product obtained after completion of process can be determined by percentage yield. Briefly powder obtained from spray drying process was collected and product yield was obtained from following equation:

$$
\text { Percentage yield }=\frac{\text { Practical yield }}{\text { Theoritical yield }} \times 100
$$

\subsubsection{Particle size analysis}

Particle size of the nanosuspension formulation was done by using particle size analyzer (Malvern Zetasizer). Size and size distribution of the nanocrystals were determined through particle size analyzer with a wet sampling system and the diameters reported were calculated using mean particle size distribution.

\subsubsection{Zeta potential (ろ) analysis}

Measurement of Zeta potential is also a prerequisite to know the stability of nanosuspension. Zeta potential is a measure of surface charge of particles and thus it imparts the colloidal stability due to particleparticle repulsion, as particle aggregation is less to occur for charged particles (a high Zeta potential). So the prediction of Zeta potential also allows the prediction of stability of nanosuspension. The zeta potential of the nano suspended particles surface was determined by electrophoretic mobility in an apparatus such as a Malvern Zetasizer (Malvern Instruments, UK) equipped with suitable software and calibrated with the supplied standard. Three consecutive measurements are performed at $25^{\circ} \mathrm{C}$ using a constant cell drive of 150 $\mathrm{mV}$. The electrophoretic mobility is converted into zeta potential values through the Smoluchowsky equation, using the dielectric constants and viscosity of dispersion medium.

\subsubsection{FTIR}

FTIR spectrum of nanocrystals reveals the fundamental peaks corresponding to the chemical nature of the drug and excipients. FTIR studies were carried out in order to determine any possible interaction among drug and excipients used. IR absorption spectrum of Hydrochlorothiazide was determined by Fourier transform infrared spectrophotometer (Bruker Alpha-T model). Spectra were recorded over the wave number 400-4000 cm-1. The FTIR spectrum of the pure drug and optimized nanocrystal formulation batch R2 was recorded. Compatibility of fibroin and drug was analyzed by overlain spectrum analysis.

\subsubsection{X-Ray diffraction studies}

This technique allows amorphous and crystalline materials to be differentiated. Crystalline materials display many diffraction bands, whereas amorphous compounds present a more or less regular baseline. The X-ray diffraction procedure to estimate the degree of crystallinity was based upon the measurement of the total scattering and the scattering from the crystalline region of formulations \& pure drug. XRD patterns of drug and fibroin were recorded at room temperature on X-ray diffractometer (Philips analytical XRD, PW $3710)$ with CuKa radiation (1.54 $\AA$ ), at $40 \mathrm{kV}, 40 \mathrm{~mA}$ and passing through a nickel filter. The diffractometer was equipped with a $2 \theta$ compensating slit and was calibrated for the accuracy of peak positions with a silicon pellet. Samples were mounted either on a $25-\mathrm{mm}$ holder made of Polymethyl methacrylate or a zero background small sample holder, depending on sample size, and were subjected to X-ray powder diffraction analysis in continuous mode with a step size of $0.01^{\circ}$ and step time of 1 second over an angular range of 3 to $40^{\circ} 2 \theta$. Sample holders were rotated in a plane parallel to their surface at $30 \mathrm{rpm}$ during the measurements.

\subsubsection{Differential scanning calorimetry}

Differential scanning calorimetry (DSC) studies were carried out using MettlerToledo DSC 821 instrument. Indium and zinc standards were used to calibrate the DSC temperature and enthalpy scale. The spray dried nanocrystals of optimized batch and pure drug Hydrochlorothiazide are hermetically sealed in 
aluminum crucibles and heated at a constant rate of $10^{\circ} \mathrm{C} / \mathrm{min}$ over a temperature range of $25-300^{\circ} \mathrm{C}$. Inert atmosphere was maintained by purging nitrogen gas at flow rate of $50 \mathrm{ml} / \mathrm{min}$. results were obtained for each sample.

\subsubsection{Percent crystallinity study}

The percentages of crystalline material, present in the samples were calculated by using equation (Rawlinson et al; 2007).

$$
\% \text { Crystallinity }=\frac{\delta H m S D}{\delta H m D r u g \times W} \times 100
$$

Where $\delta H m S D$ is the melting enthalpy of the solid dispersion $\left(\mathrm{Jg}^{-1}\right), \delta H m D r u g$ is the melting enthalpy of drug $\left(\mathrm{Jg}^{-1}\right)$ and $\mathrm{W}$ is the weight fraction of drug in fibroin.

\subsubsection{Percent drug content}

The spray dried nanocrystal powder $(10 \mathrm{mg})$ was dissolve in $1 \mathrm{ml}$ methanol and volume was made up to mark in $10 \mathrm{ml}$ volumetric flask with phosphate buffer $\mathrm{pH}$ 6.8. $0.1 \mathrm{ml}$ of above solution was further diluted to $10 \mathrm{ml}$ and analyzed by spectrophotometrically at $274 \mathrm{~nm}$. The Hydrochlorothiazide content in nanocrystals $(\% \mathrm{w} / \mathrm{w})$ was calculated using calibration curve.

\subsubsection{In-vitro dissolution study}

To understand the characteristics of drug dissolution from nanocrystals, an in vitro dissolution was carried out. For investigating better dissolution profile of batches, dissolution studies were performed for the nanocrystal formulations.

Preparation of dissolution medium

Acid buffer $p H 1.2$

The dissolution medium used for in vitro dissolution study was acidic buffer $\mathrm{pH} 1.2$ containing $250 \mathrm{ml}$ potassium chloride in $425 \mathrm{ml}$ of $0.2 \mathrm{M} \mathrm{HCL}$ and made volume upto $1000 \mathrm{ml}$ with sufficient distilled water.

\section{Phosphate buffer $p H 6.8$}

Another dissolution medium used for in vitro dissolution study was phosphate buffer $\mathrm{pH}$ 6.8containing $28.80 \mathrm{gm}$ of disodium hydrogen phosphate and 11.45 of potassium dihydrogen phosphate dissolved in sufficient water and volume made upto $1000 \mathrm{ml}$.

Procedure for in vitro drug dissolution study

In vitro dissolution of Hydrochlorothiazide from nanocrystals was evaluated by the basket apparatus. The dissolution medium consists of $\mathrm{pH} 6.8$ phosphate buffer. The nanocrystals was dissolved in buffer and was placed in the basket. The dissolution test was performed in USP type I dissolution apparatus I (Electrolab) according to United State Pharmacopoeia dissolution procedure. The nanocrystals of all batches were filled into hard gelatin capsules. The HCTZ nanocrystal hard gelatin capsule put into basket which containingthe $900 \mathrm{ml}$ of acidic buffer $\mathrm{pH} 1.2$ and phosphate buffer at $37 \pm 0.5^{\circ} \mathrm{C}$ with speed of $100 \mathrm{rpm}$. Each sample (5 ml) was withdrawn at $15,30,45,60 \mathrm{~min}$ and $1,2,3,4,5,6,7,8,9,10,11,12 \mathrm{~h}$ respectively with replacement by an equal volume of temperature equilibrated media and filtered through $0.45 \mu \mathrm{m}$ pore size nylon filter. The amount of drug dissolved determined by UV spectroscopy at $\lambda \max$ of $274 \mathrm{~nm}$ for acidic buffer $\mathrm{pH} 1.2$ and phosphate buffer $\mathrm{pH} 6.8$ [9].

\subsubsection{Scanning electron microscopy}

Evaluation of particle size and morphology was achieved via scanning electron microscopy (SEM), using a JSM-6360 microscope (JEOL, Tokyo, Japan). Samples were scattered on a thin film of a two component epoxy resin and then coated with a platinum layer. Acceleration during the observation was 250, 500, 1000 and $2000 \mathrm{kV}$.

\subsubsection{Stability study}

For effective stabilization and a reasonable processing time, strong and fast adsorption is necessary, with full coverage and slow desorption. [14] The intention behind the stability testing was carry out under the 
stress conditions and effect of factors such as light, temperature, humidity on the drug or formulations. From that, we can estimate the shelf life of the drug or formulation at different storage conditions.

Batch R2 has selected as a representative for accelerated stability study. Drug content, particle size, zeta potential \& crystallinity studies performed for batch R2 at different time intervals. This batch packed individually in zip lock pouch covered with aluminum foil. Accelerated stability study has carried out at two different conditions, for 3 months period at temperature $2-8^{\circ} \mathrm{C}$ and RT.

Sampling done at the interval of 30th day, 60th day, and 90th day under previously mentioned conditions.

Author contributions: Concept - R.D., U.P., N.J.; Design - R.D., U.P., N.J.; Supervision - R.D., U.P., N.J.; Data Collection and/or Processing - R.D.; Analysis and/or Interpretation - R.D., U.P., N.J.; Literature Search - R.D., N.J.; Writing - R.D.; Critical Reviews - R.D., U.P., N.J.

Conflict of interest statement: The authors received no financial support for the research, authorship, and/or publication of this article. The authors declare no conflict of interest.

\section{REFERENCES}

[1] Katteboina S, Chandrasekhar P, Balaji S. Drug nanocrystals: a novel formulation approach for poorly soluble drugs. Int J Pharm Tech Res. 2009; 1(3): 682-694.

[2] Patravale V, Date A, Kulkarni R. Nanosuspensions a promising drug delivery strategy. J Pharm Pharmacol. 2004; 56: 827-840. [CrossRef]

[3] Kale M,Mishra B. Drug nanocrystals: A way toward scale-up. Saudi Pharm J. 2016; 24(4): 386-404.[CrossRef]

[4] Pawar V,Singh Y, Meher J, Gupta S, Chourasiya M. Engineered nanocrystal technology: in-vivo fate, targeting and applications in drug delivery. J Control Release. 2014; 183: 51-66. [CrossRef]

[5] Buraphacheep V, Morakul B. Nanocrystals for enhancement of oral bioavailability of poorly water-soluble drugs. Asian J Pharm Sci. 2015; 13-23. [CrossRef]

[6] Wei H, Ye L, Jianping Q, Lingyun C, Fuqiang H, Wei W. Food proteins as novel nanosuspension stabilizers for poorly water-soluble drugs. Int J Pharm. 2013; 441: 269-278. [CrossRef]

[7] Cheng Y, Koh LD, Li D, Ji B, Hang MY, Zhang YW. On the strength of $\beta$-sheet crystallites of Bombyx mori silk fibroin. J R Soc Interface. 2014; 11: 1-9. [CrossRef]

[8] Bajaj A, Rao M, Pardeshi A, Sali D. Nanocrystallization by Evaporative Antisolvent Technique for Solubility and Bioavailability Enhancement of Telmisartan. AAPS Pharm Sci Tech. 2012; 13(4): 1331-1340. [CrossRef]

[9] Nanjwade B, Derkar G, Bechra H,Nanjwade V, Mani F. Design and Characterization of Nanocrystals of Lovastatin for Solubility and Dissolution Enhancement. J Nanomed Nanotechnol. 2011; 2: 107. [CrossRef]

[10] Choi M, Briançon S, Andrieu J, Min S, Fessi H. Effect of freeze drying process conditions on the stability of nanoparticles. Dry Technol. 2004; 22: 335-346. [CrossRef]

[11] Kakran M, Shegokar R, Sahoo NG, Shaal LA, Li L, Muller RH. Fabrication of quercetin nanocrystals: comparison of different methods. Eur J Pharm Biopharm. 2010; 80: 113-121. [CrossRef]

[12] Lee J, Lee SJ, Choi JY, Yoo JY, Ahn CH. Amphiphilic amino acid copolymers as stabilizers for the preparation of nanocrystal dispersion. EurJ Pharm Sci 2005; 24(5): 441-449. [CrossRef]

[13] Patel AP, Patel JK, Patel KS, Deshmukh AB, Mishra BR. A review on drug nanocrystal a carrier free drug delivery. Int J Res Ayurveda Pharm. 2(2), 448-458 (2011).

[14] Peltonen, L. Hirvonen J. Pharmaceutical nanocrystals by nanomilling: critical process parameters, particle fracturing and stabilization methods. J Pharm Pharmacol. 2010; 62(11): 1569-1579. [CrossRef] 